\section{Sodium hyaluronate eye drops prevent late-onset bleb leakage after trabeculectomy with mitomycin C}

H Sagara', ${ }^{1,2}$, T lida², K Suzuki2,3, T Fujiwara ${ }^{2}$, $\mathrm{H} \mathrm{Koizumi}^{2}$ and $\mathrm{K}$ Yago $^{2}$

\section{Abstract}

Purpose To evaluate the efficacy of sodium hyaluronate eye drops for prevention of late-onset bleb leakage after trabeculectomy with mitomycin C (MMC).

Methods Sixty-eight patients (68 eyes) who had trabeculectomy with MMC from March 1995 to February 2002 at the Marui Eye Clinic or the Department of Ophthalmology, Fukushima Medical University Hospital were examined in this study. Twenty-two were treated postoperatively with sodium hyaluronate eye drops (sodium hyaluronate group) and 46 eyes were not treated postoperatively (control group). The blebs were classified according to the avascular area, epithelial defect and degree of bleb wall leakage. Both groups were assigned classes (0, avascular or partial avascular bleb; 1 , avascular or partial avascular bleb with an epithelial defect; 2 , avascular or partial avascular bleb with oozing; 3 , avascular or partial avascular bleb with a point of leakage). Results Follow-up duration ranged from 10 to 116 months (mean \pm SD, $57.6 \pm 30.5$ ). The incidence of reclassification from class 1 to class 0 was significantly greater in the sodium hyaluronate group (four of six class 1 eyes improved to class 0 after application of sodium hyaluronate eye drops) than in the control group (zero of 10 eyes) $(P=0.008)$. And, the incidence of reclassification from class 1 to class 2 or class 3 was significantly smaller in the sodium hyaluronate group (one of six eyes) than in the control group (nine of 10 eyes) $(P=0.008)$. Conclusions Sodium hyaluronate eye drops may prevent late-onset bleb leakage after trabeculectomy with MMC.

Eye (2008) 22, 507-514; doi:10.1038/sj.eye.6702674; published online 15 December 2006
Keywords: trabeculectomy; mitomycin C; sodium hyaluronate; bleb; leakage

\section{Introduction}

As the introduction of 5-fluorouracil (5-FU) and mitomycin C (MMC) in glaucoma filtering surgery, the incidence of late-onset bleb leaks has increased. ${ }^{1-3}$ Bleb leaks may lead to hypotony maculopathy, choroidal detachment, blebitis and endophthalmitis. $^{2-6}$ Various methods of both medical and surgical management of late-onset bleb leaks exist, such as bandaged contact lenses, ${ }^{7}$ autologous blood injections ${ }^{8}$ and leaking bleb reconstruction with rotational or advancement conjunctival flap, ${ }^{9}$ but none are without difficulty or have been universally successful; and in fact, treatments have often been associated with complications. Surgical bleb revision can cause immediate postoperative intraocular pressure elevations, persistent or recurrent leaks, conjunctiva shrinkage and retraction, wound dehiscence and additional filtering surgery. ${ }^{10}$ Therefore, a medical method of prevention of late-onset bleb leaks is needed.

Thin avascular blebs resulting from trabeculectomy with MMC frequently lack conjunctival epithelium. ${ }^{11,12}$ We also reported that epithelial failure of the bleb wall, caused by tear dysfunction, could contribute to the occurrence of late-onset bleb leakage after trabeculectomy with MMC, and dry eye is a risk factor of late-onset bleb leakage. ${ }^{13}$

The topical application of autologous serum has been reported to improve

keratoconjunctival dysfunction, effectively treat severe dry eye ${ }^{14}$ and to seal late-onset bleb leaks. ${ }^{15,16}$ However, this treatment requires collection of blood from the patients and is difficult to perform over a long period of time.
${ }^{1}$ The Marui Eye Clinic, Minamisouma, Fukushima, Japan

${ }^{2}$ Department of Ophthalmology, Fukushima Medical University School of Medicine, Fukushima, Japan

${ }^{3}$ Ohara General Hospital, Fukushima, Japan

Correspondence: H Sagara, Marui Eye Clinic, 1-83-3, Honjinmae Haramachi-ku, Minamisouma, Fukushima 975-0062, Japan

Tel: + 8124424 0101; Fax: + 81244240148

E-mail: hide1234@ ruby.ocn.ne.jp

Received: 5 May 2006 Accepted in revised form: 30 October 2006

Published online: 15 December 2006 
Although the effectiveness of sodium hyaluronate eye drops treatment for late-onset bleb leakage has not been widely reported, it is an efficient and simple treatment for dry eye. ${ }^{17}$ In the current study, the therapeutic efficacy of sodium hyaluronate eye drops for the prevention of late-onset bleb leakage was prospectively evaluated in glaucoma patients who had undergone trabeculectomy with MMC.

\section{Patients and methods}

The patients underwent trabeculectomy with MMC from March 1995 to February 2002 at the Marui Eye Clinic or the Department of Ophthalmology, Fukushima Medical University Hospital. The study adhered to the tenets of the Declaration of Helsinki Principles. Treatments were administered after the purpose of the study was explained to the patients and they were provided informed consent. Doctors explained that sodium hyaluronate eye drops effectively treat dry eye and rarely cause severe side effects. Moreover, the patients were told that the sodium hyaluronate eye drops prevent complications of trabeculectomy with MMC.

Patients with dry eye appear to be at high risk for lateonset bleb leakage. ${ }^{13}$ However, diagnosis of dry eye is difficult, as it can be caused by various diseases. ${ }^{18}$ Therefore, two patients (two eyes) with severe corneal epithelial failure and tear dysfunction were excluded from this study. Moreover, seven patients (seven eyes) who used artificial tears after trabeculectomy were excluded from the study because it is an efficacious drop to dry eye. Some patients received trabeculectomy in both eyes, but only one eye was included in the study. When trabeculectomy was performed two or more times in a patient, the patient was included in the study after the last procedure was completed. These patients were documented as having undergone a previous incisional operation.

In the remaining 69 patients (69 eyes), 23 eyes (23 patients) received topical postoperative application of $0.1 \%$ sodium hyaluronate eye drops (Hyalein, Santen Pharmaceutical Co. Ltd.) (sodium hyaluronate group); the remaining 46 eyes (46 patients) were not treated with sodium hyaluronate eye drops postoperatively (control group). The doctors were instructed to initiate application of sodium hyaluronate eye drops immediately or 1 year after the procedure. Patients who stopped sodium hyaluronate application were included in this study only when sodium hyaluronate had been administered for more than 6 months. One patient (one eye) who used sodium hyaluronate for less than 6 months, owing to discomfort, was excluded. Ultimately, 68 patients (68 eyes) were enrolled in this study (22 eyes in the sodium hyaluronate group and 46 eyes in the control group). Only two patients (two eyes) were treated at the Marui Eye Clinic and 66 patients (66 eyes) were treated at the Department of Ophthalmology, Fukushima Medical University Hospital.

Patient demographics, follow-up duration, glaucoma diagnosis, history of previous incisional operation, and bleb type were recorded.

\section{Method of assigning eyes}

The patients were assigned to the two groups just before surgery by their outpatient clinic according to the day of the week. Permuted block design with the table of random numbers was used to assign the protocol of application of sodium hyaluronate eye drops immediately or 1 year after the procedure in the proportion of one to two. In addition, complete randomization with the table of random numbers was used to assign the left or right eye when the procedure was performed in both eyes. The patients from both centers were assigned individually.

\section{Method of trabeculectomy with MMC}

A 67-mm limbus-based conjunctival flap was created superiorly or inferiorly and a $4 \times 4$-mm square, one-third to one-half thick scleral flap was created. Small pieces of surgical sponges soaked in $0.04 \% \mathrm{MMC}$ were placed under the conjunctival flap for 3-5 min. After the sponges were removed, the area was irrigated immediately with $200 \mathrm{ml}$ of saline solution. At the edge of the corneoscleral bed, a block of clear corneal and trabecular meshwork tissue was removed and a peripheral iridectomy was performed. The scleral flap was closed with five to seven monofilament 10-0 nylon interrupted sutures. The Tenon capsule in the area of the conjunctival incision was excised, but no attempt was made to remove all of the Tenon capsule. The conjunctival flap and the underlying Tenon's capsule were closed with running or interrupted 10-0 nylon sutures.

\section{Classification of late-onset bleb leakage}

The frequency of late-onset bleb leakage and time of leakage vary, and are affected by the surgical technique. ${ }^{1,2,4,5}$ Belyea et $a l^{2}$ reported that late-onset bleb leaks occurred in seven eyes (six patients) out of 385 eyes (304 patients) more than 6 months after trabeculectomy with 5-FU or MMC, using a technique very similar to our method. In addition, to minimize the effect of the procedure, we defined in this study late-onset bleb leakage as bleb leakage beginning more than 6 months after trabeculectomy with MMC. 

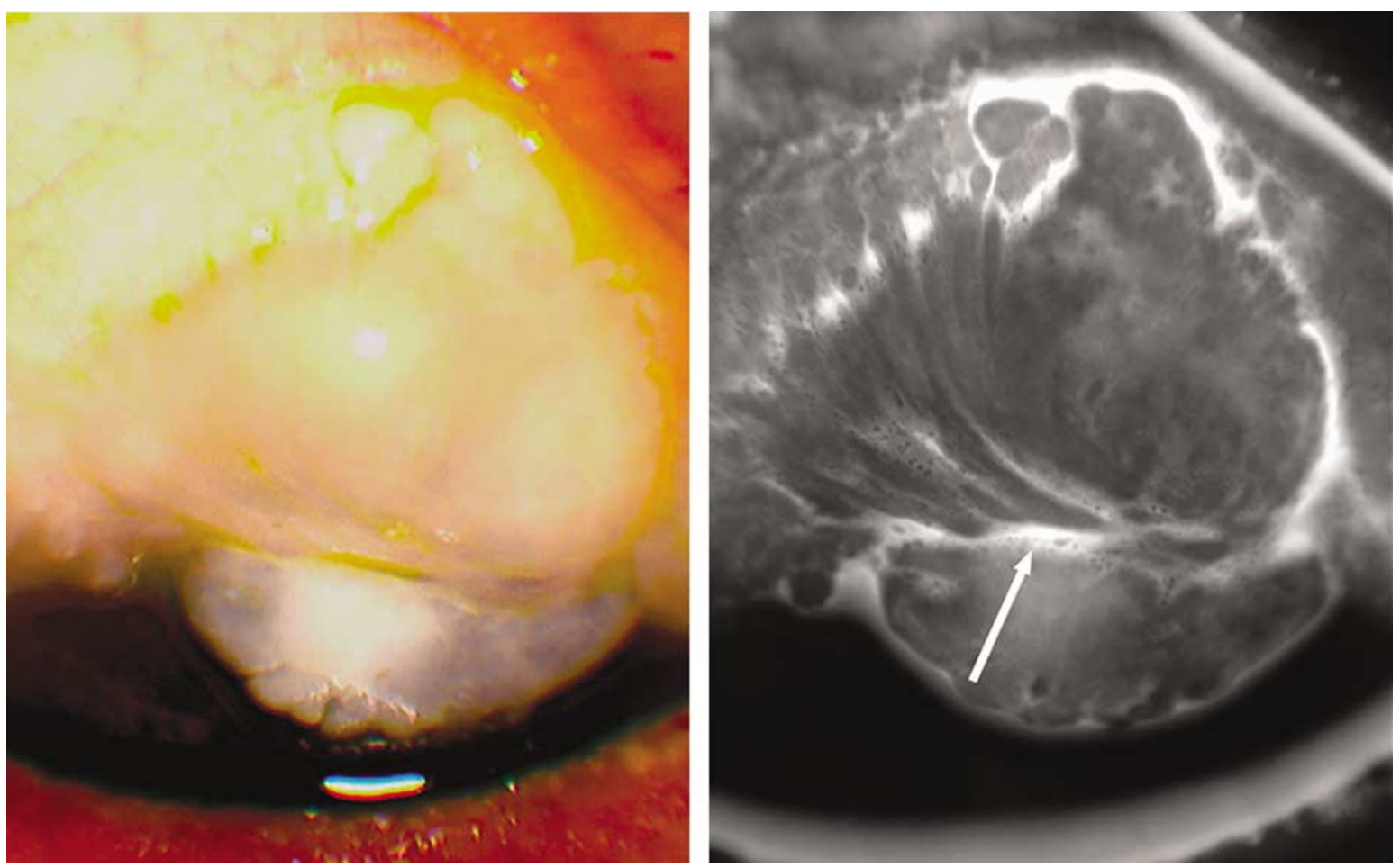

Figure 1 Class 0. A 70-year-old man. Trabeculectomy with MMC had been performed 4 years earlier. Sodium hyaluronate eye drops were applied to his class 0 eyes during the previous 3 years. (Left) Color photograph taken with a fundus camera (TRC-50LX, Topcon, Tokyo, Japan) of the anterior segment. The bleb is type 1 and avascular with a partial thin wall. (Right) Anterior segment photograph taken with a fundus camera in the fluorescein angiogram mode. Although the bleb wall is not smooth and some microcysts are observed (arrow), no epithelial defect is detected.

The blebs were classified based on the following characteristics. ${ }^{19,20}$ Type 1 blebs had good filtration and were elevated with a relatively avascular appearance compared with the surrounding conjunctiva. Type 2 blebs had good filtration and were diffuse with a relatively avascular appearance compared with the surrounding conjunctiva. Flat blebs had poor filtration with flat and engorged surface blood vessels.

Encapsulated blebs had poor filtration and a localized, highly elevated, fluid-filled cavity of hypertrophied Tenon capsules with engorged surface blood vessels.

Two types of aqueous leakage have been reported, ${ }^{4,21}$ that is, point leaks and oozing from avascular blebs. Point leaks have a visible aqueous stream from a leakage point; oozing is characterized by transconjunctival aqueous egress without a focal leakage point. In this study, all patients who developed oozing had an untreated epithelial defect of the bleb wall without apparent penetration of aqueous humor, and oozing progressed to a point leak. Furthermore, as epithelial failure and the avascular area of the bleb wall are reportedly associated with late-onset bleb leakage, ${ }^{2,13}$ the blebs were classified as follows: class 0 , avascular or partial avascular bleb (Figure 1); class 1, avascular or partial avascular bleb with an epithelial defect; class 2 , avascular or partial avascular bleb with oozing (Figure 2); and class 3, avascular or partial avascular bleb with a point leak.

\section{Evaluation of epithelial defects and bleb leaks}

A moistened sterile fluorescein strip was gently applied to the palpebral conjunctiva of the lower eyelid to prevent bleb damage. Epithelial failure of the bleb wall, oozing or a spontaneous visible aqueous stream from a leakage point, was determined by examination with a slit-lamp, using cobalt blue illumination after two or three eye blinks. If the epithelial condition was not observed clearly, a fundus camera in fluorescein angiogram mode was used. This technique can separate exciting light from emitted light and the epithelial failure can be examined in high contrast using appropriate filters. ${ }^{22}$ When epithelial failure was observed, but points of leakage were not found, the slit-lamp observation was continued for $10 \mathrm{~s}$ to locate the oozing area.

The epithelial defect of a bleb wall detected more than 6 months after trabeculectomy with MMC was considered as 'epithelial defect' to minimize the influence of the procedure. 

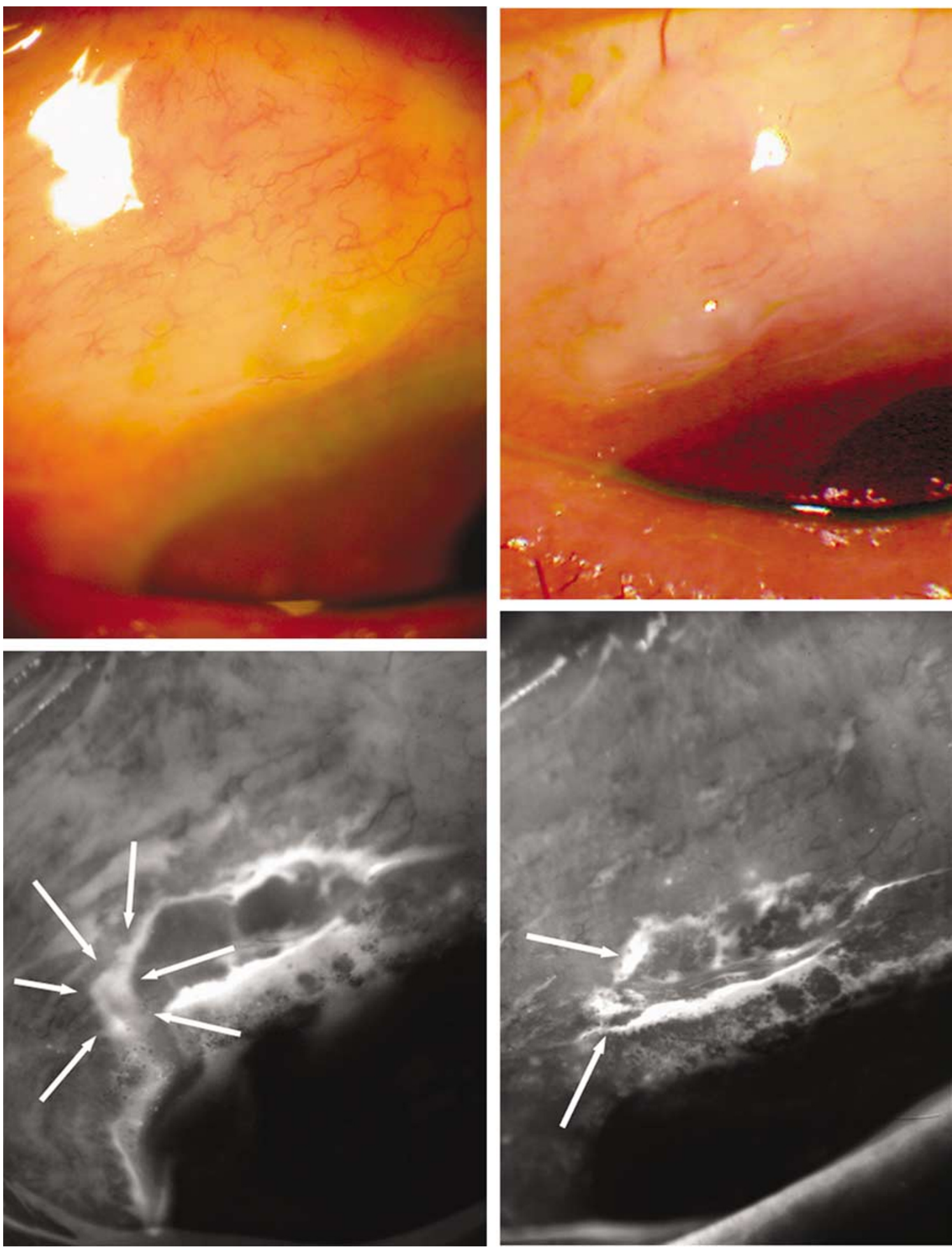

Figure 2 Class 2. A 57-year-old man. Trabeculectomy with MMC was performed 8 years earlier. Oozing was detected 6 years after trabeculectomy with MMC. (Top left and right) The bleb is type 2, thin-walled, and partially avascular with oozing and an epithelial defect (arrows) before application of sodium hyaluronate eye drops. (Bottom left and right) Although the epithelial defect and oozing resolved 1 month after application of sodium hyaluronate eye drops began, microcysts (arrows) are detected on the surface of the bleb wall. 


\section{Diagnosis of corneal epithelial failure and tear dysfunction}

Corneal epithelial failure was determined by fluorescein staining of the ocular surface. Epithelial failure observed over more than $2 / 3$ of the corneal surface was considered severe.

Tear dysfunction was determined by the Schirmer test using topical anesthesia induced with $0.4 \%$ oxybuprocaine hydrochloride, and by tear break-up time (BUT) and the Cotton thread test. The Schirmer test was performed after the conjunctival sac was wiped. Tear examinations were performed more than 6 months after trabeculectomy with MMC to minimize the effect of the procedure.

Patients with a 5-mm or less Schirmer test result, a 5-second or less BUT, or a $10-\mathrm{mm}$ or less Cotton thread test result were diagnosed with tear dysfunction. ${ }^{18,23}$ Rose Bengal staining of the ocular surface was not performed owing to its toxicity to the keratoconjunctival epithelial cells. ${ }^{24}$

\section{Patient follow-up}

All patients were treated with $0.1 \%$ fluorometholone or $0.1 \%$ betamethasone sodium phosphate eye drops and $0.3 \%$ ofloxacin eye drops four times daily for 3 months after the surgery. Elevated intraocular pressure after surgery was treated topically with beta-blockers, prostaglandin-related agents and/or carbonic anhydrase inhibitors. Patients were instructed to visit the outpatient clinic every 2 weeks or once a month and to instill the sodium hyaluronate eye drops four to six times daily while supine so the drops could spread onto the upper blebs.

The patients in the sodium hyaluronate group were instructed to use the eye drops from the initial application to the last examination. The doctors verified with the patients at every visit that sodium hyaluronate eye drops had been administered.

\section{Statistical analyses}

Group differences for continuous variables were assessed with the unpaired $t$-test. Differences for categorical variables were assessed with the $\chi^{2}$ test or Fisher's exact test for independence. A $P$-value of $<0.05$ was considered statistically significant.

All statistical analyses were performed using the JMP professional edition for Windows (Release 5.01a, SAS Inc., Cary, NC, USA).

\section{Results}

One eye of the sodium hyaluronate group and three eyes of the control group were treated with trabeculectomy with MMC by an inferior approach. Late-onset leakage (class 3) was detected in only one eye treated with the inferior approach in the control group. Other eyes were treated with a superior approach.

The follow-up time from trabeculectomy with MMC to the last examination ranged from 10 to 116 months for all eyes (mean $\pm S D ; 57.6 \pm 30.5$ months). The two study groups did not differ significantly with regard to age, sex, length of follow-up, glaucoma diagnosis, history of previous incisional operation or bleb type detected at the last examination (Table 1).

In the sodium hyaluronate group, when reepithelialization occurred on a defective epithelial area of the bleb wall, microcysts were detected on the bleb surface (Figure 2). When the bleb wall of the defective epithelial area thickened, the microcysts disappeared.

Figure 3 shows the variations in the bleb classifications after trabeculectomy with MMC with or without subsequent administration of sodium hyaluronate eye drops.

\section{Outcome of treatment with sodium hyaluronate eye drops immediately after trabeculectomy with MMC}

Ten $(21.7 \%)$ of 46 eyes in the control group became flat or encapsulated without being reclassified into class 0 ,

Table 1 Demographics and clinical characteristics of the sodium hyaluronate and control groups

\begin{tabular}{lccc}
\hline & $\begin{array}{c}\text { Sodium } \\
\text { hyaluronate } \\
\text { group }\end{array}$ & Control group & $\mathrm{P}^{*}$ \\
& 22 & & \\
\hline No. eyes & $65.2 \pm 10.6$ & $60.5 \pm 17.5$ & $0.253^{\mathrm{b}}$ \\
Age (yrs) & $16 / 6$ & $24 / 22$ & $0.123^{\mathrm{c}}$ \\
Male/female & $55.0 \pm 29.0$ & $58.8 \pm 31.4$ & $0.626^{\mathrm{b}}$ \\
Follow-up (mos) & & & $0.801^{\mathrm{d}}$ \\
Diagnosis & 13 & 29 & \\
POAG, NTG, PXS & 2 & 5 & \\
PACG, CACG & 7 & 10 & \\
Secondary glaucoma & 0 & 1 & \\
Congenital glaucoma & 0 & 1 & \\
Developmental glaucoma & 5 & 10 & $1.000^{\mathrm{c}}$ \\
Previous incisional surgery & $5 / 12$ & $9 / 19$ & $1.000^{\mathrm{c}}$ \\
Total (type 1/type 2) & $4 / 11$ & $4 / 14$ & \\
Class 0 (type1/type 2) & $0 / 1$ & $0 / 1$ & \\
Class 1 (type1/type 2) & $1 / 0$ & $1 / 2$ & \\
Class 2 (type1/type 2) & - & $4 / 2$ & \\
Class 3 (type1/type 2) & & &
\end{tabular}

POAG = primary open-angle glaucoma; $\mathrm{NTG}=$ normal tension glaucoma; PXS = pseudoexfoliation syndrome; PACG $=$ primary angle-closure glaucoma; $\mathrm{CACG}=$ chronic angle-closure glaucoma.

${ }^{*} P=P$-value of null hypothesis. There is no difference between the sodium hyaluronate and control groups.

${ }^{\mathrm{a}}$ Mean \pm SD

bUnpaired $t$-test.

'Fisher's exact test.

${ }^{\mathrm{d}} \chi^{2}$ test.

${ }^{\text {Thpe }} 1$ blebs had an elevated bleb with a relative avascular appearance. Type 2 blebs were diffuse with a relative avascular appearance. 


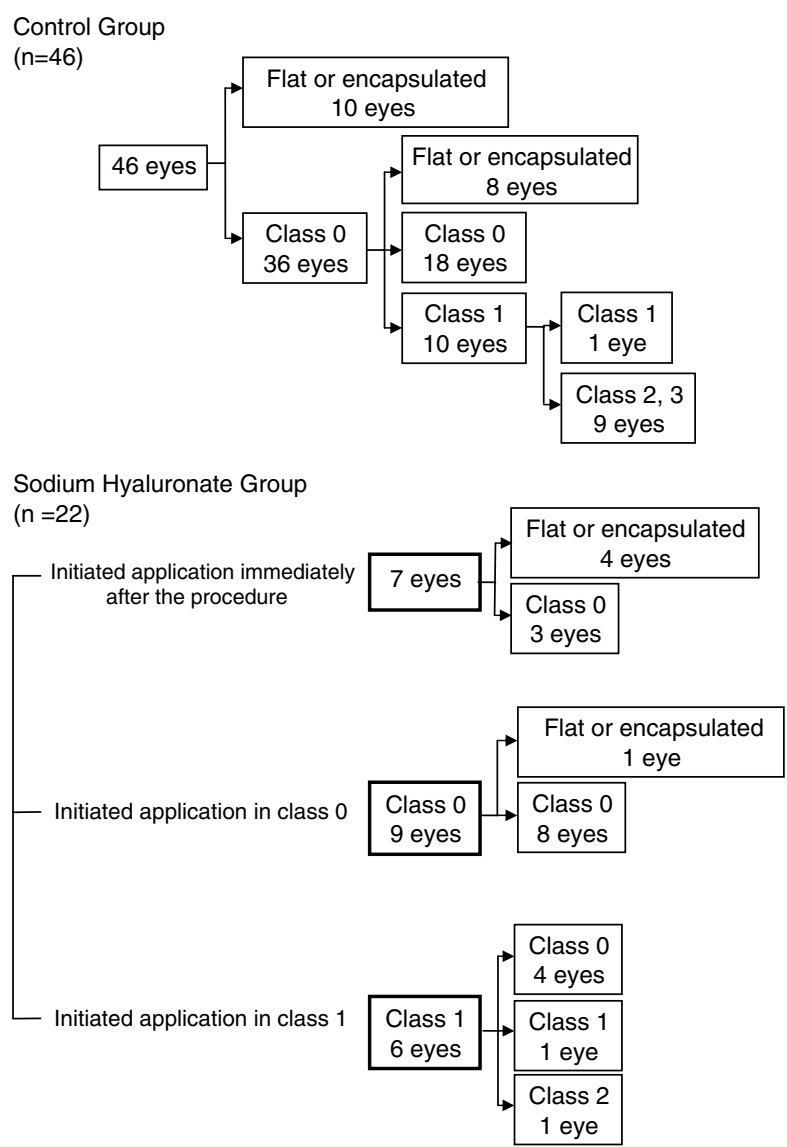

Figure 3 Comparison of the variation in late-onset leaking bleb classification after trabeculectomy with MMC with or without subsequent sodium hyaluronate treatment. In the control group eyes, the changes in the classes of the blebs reflect the natural changes. In the sodium hyaluronate group eyes, the changes in the bleb classification result from initiation of the topical application of sodium hyaluronate eye drops immediately or 1 year after the procedure, as outlined in bold.

whereas four $(57.1 \%)$ of seven eyes became flat or encapsulated in the sodium hyaluronate group. Thirtysix $(78.3 \%)$ of 46 eyes were reclassified into class 0 in the control group, whereas three $(42.9 \%)$ of seven eyes were reclassified into class 0 in the sodium hyaluronate group.

The incidence of immediate flattening or encapsulation tended to be greater in the sodium hyaluronate group (four of seven eyes) than in the control group (10 of 46 eyes), although the difference was not statistically significant $(P=0.070)$. Reclassification of blebs into class 0 tended to be less likely to occur in the sodium hyaluronate group (three of seven eyes) than in the control group (36 of 46 eyes), although the difference was not statistically significant $(P=0.070)$.

\section{Outcome of class 0 eyes after treatment with sodium hyaluronate eye drops}

In the control group, eight (22.2\%) of 36 class 0 eyes became flat or encapsulated. In the sodium hyaluronate group, one of nine class 0 eyes became flat or encapsulated. Ten (27.8\%) of 36 class 0 eyes in the control group were reclassified into class 1 , whereas no class 0 eyes in the sodium hyaluronate group were reclassified into class 1.The incidence of flattening or encapsulation from class 0 did not differ significantly between the sodium hyaluronate (one of nine eyes) and control groups (eight of 36 eyes) $(P=0.661)$. The incidence of reclassification from class 0 to class 1 did not differ significantly between the sodium hyaluronate (zero of nine eyes) and control groups (10 of 36 eyes) $(P=0.173)$.

\section{Outcome of class 1 eyes after treatment with sodium hyaluronate eye drops}

In the control group, none of the 10 class 1 eyes were reclassified into class 0 (epithelial defect of the bleb wall was not improved in any eyes). In the sodium hyaluronate group, four of six class 1 eyes were reclassified into class 0 . In the control group, nine of 10 class 1 eyes were reclassified into class 2 or class 3 . In the 
sodium hyaluronate group, one of six class 1 eyes was reclassified into class 2 .

The incidence of reclassification from class 1 to class 0 was significantly greater in the sodium hyaluronate group (four of six eyes) than in the control group (zero of 10 eyes) $(P=0.008)$. The incidence of reclassification from class 1 to class 2 or class 3 was significantly smaller in the sodium hyaluronate group (one of six eyes) than in the control group (nine of 10 eyes) $(P=0.008)$. None of the eyes developed blebitis or endophthalmitis.

\section{Discussion}

A number of investigators have presented various useful classifications of filtering blebs with morphological criteria such as size of the avascular area, thickness of the bleb wall and degree of the leakage..$^{3,4,19,20}$ In addition, epithelial defects can be detected before apparent leakage occurs from the bleb wall. Therefore, we used a new classification of the late-onset bleb leakage: avascular or partial avascular bleb with an epithelial defect.

The incidence of immediate flattening or encapsulation did not differ significantly between the control group and the group administered sodium hyaluronate eye drops immediately after trabeculectomy with MMC. However, the incidence of flattening or encapsulation tended to be greater in the sodium hyaluronate group, with over $50 \%$ of the eyes becoming flat or encapsulated. Sodium hyaluronate eye drops may advance fibrosis of the bleb wall and decrease bleb function.

When sodium hyaluronate eye drops were administered immediately after trabeculectomy with $\mathrm{MMC}$, no eyes were reclassified into class 1 . In addition, when sodium hyaluronate eye drops were administered to class 0 eyes, no eyes were reclassified into class 1 . The incidence of reclassification from class 1 to class 0 was greater in the sodium hyaluronate group. Furthermore, the incidence of reclassification from class 1 to class 2 or class 3 was smaller in the sodium hyaluronate group. Therefore, sodium hyaluronate eye drops may prevent and improve epithelial defects of the bleb wall.

When the defective epithelial area of the bleb wall improved, microcysts were detected on the bleb surface. Similar microcysts, which occur on the surface of the cornea with bullous keratopathy in many cases, ${ }^{25}$ are formed as a result of stromal edema of the cornea. Microcysts are often detected on functional thin bleb surfaces (Figure 1). Stromal edema of the conjunctiva, owing to intra-bleb aqueous humor, appears to have induced microcysts with thin conjunctival epithelium. Therefore, the microcysts appeared with reepithelialization of the bleb wall because the leakage from the defective epithelial area had been suppressed owing to the thin conjunctival epithelium.
Although the effects of sodium hyaluronate eye drops on the conjunctiva have not been studied fully, sodium hyaluronate reportedly enhances re-epithelialization of the conjunctiva by suppressing break-up of the preocular tear film. ${ }^{26}$ The tear fluid, which contains various cytokines, such as epidermal growth factor and vitamin A, helps maintain the normal histology of the conjunctiva. ${ }^{27,28}$ In the current study, the tear fluid, which suppressed the break-up by sodium hyaluronate eye drops, might have enhanced fibrosis and reepithelialization of the bleb wall. Moreover, sodium hyaluronate stimulates corneal epithelial migration and accelerates healing of corneal epithelial defects. ${ }^{29}$ Thus, sodium hyaluronate may stimulate epithelial migration of the bleb surface and accelerate healing of bleb surface epithelial defects.

The number of patients in this study treated with the inferior approach was small and evaluation of the risk of infection of the bleb treated with the inferior approach was difficult. However, as Higginbotham et $a l^{30}$ suggested, inferior trabeculectomy performed with MMC carries a significantly increased risk of bleb-related endophthalmitis, possibly because chronic mechanical irritation by the lower lid margin from repetitive blinking and eye movements weakens the structural integrity of an already thin, cystic bleb surface. Additional disruption of the epithelial surface, secondary to prolonged interpalpebral exposure and drying, may further alter the natural protective function of the conjunctiva, allowing increased access to the intraocular cavity of potential pathogens present on the lid margin and in the tear lake.

As Lehmann et $a l^{6}$ suggested, such thin and avascular bleb walls with denuded epithelium and underlying fullthickness stromal damage, detected after trabeculectomy with MMC, are probably less effective physical barriers against bacteria. These areas compromise the conjunctival contribution to the ocular innate immune response and have a high risk of complications by infection. ${ }^{31}$ Accurate diagnosis and treatment of epithelial defects of the bleb wall before apparent bleb leaks should reduce the risk of future bleb-related complications, such as bleb leakage, blebitis, and endophthalmitis.

Pang et $a l^{32}$ detected tear film abnormality after trabeculectomy with 5-FU, but not following treatment with MMC. Additionally, Matsuo et $a l^{4}$ found that oozing was significantly more common after treatment with 5-FU than MMC. Therefore, tear dysfunction should be carefully examined after trabeculectomy with 5-FU in order to prevent late-onset bleb leaks.

In conclusion, careful observation of the bleb surface is important. Upon detection of epithelial failure on the bleb surface, a slit-lamp examination with cobalt blue 
illumination or a fundus camera in fluorescein angiogram mode should be performed to evaluate the bleb surface condition. Appropriate use of sodium hyaluronate eye drops in class 0 or class 1 cases may prevent the development of late-onset bleb leaks. However, if flattening or encapsulation is observed, sodium hyaluronate eye drops should be stopped immediately in order to maintain bleb function.

Patients with severe corneal epithelial failure and tear dysfunction were excluded from this study. However, it is necessary to discuss how to deal with severe dry eye in the future, as dry eye is a risk factor for late-onset bleb leaks.

\section{References}

1 The Fluorouracil Filtering Surgery Study Group. Five-year follow-up of the Fluorouracil Filtering Surgery Study. Am J Ophthalmol 1996; 121: 349-366.

2 Belyea DA, Dan JA, Stamper RL, Lieberman MF, Spencer WH. Late onset of sequential multifocal bleb leaks after glaucoma filtration surgery with 5-fluorouracil and mitomycin C. Am J Ophthalmol 1997; 124: 40-45.

3 Azuara-Blanco A, Katz LJ. Dysfunctional filtering blebs. Surv Ophthalmol 1998; 43: 93-126.

4 Matsuo H, Tomidokoro A, Suzuki Y, Shirato S, Araie M. Late-onset transconjunctival oozing and point leak of aqueous humor from filtering bleb after trabeculectomy. Am J Ophthalmol 2002; 133: 456-462.

5 Greenfield DS, Liebmann JM, Jee J, Ritch R. Late-onset bleb leaks after glaucoma filtering surgery. Arch Ophthalmol 1998; 116: 443-447.

6 Lehmann OJ, Bunce C, Matheson MM, Maurino V, Khaw $\mathrm{PT}$, Wormald $\mathrm{R}$ et al. Risk factors for development of posttrabeculectomy endophthalmitis. Br J Ophthalmol 2000; 84: 1349-1353.

7 Blok MD, Kok JH, van Mil C, Greve EL, Kijlstra A. Use of the Megasoft Bandage Lens for treatment of complications after trabeculectomy. Am J Ophthalmol 1990; 110: 264-268.

8 Leen MM, Moster MR, Katz LJ, Terebuh AK, Schmidt CM, Spaeth GL. Management of overfiltering and leaking blebs with autologous blood injection. Arch Ophthalmol 1995; 113: 1050-1055.

9 O'Connor DJ, Tressler CS, Caprioli J. A surgical method to repair leaking filtering blebs. Ophthalmic Surg 1992; 23: 336-338.

10 Feldman RM, Altaher G. Management of late-onset bleb leaks. Curr Opin Ophthalmol 2004; 15: 151-154.

11 Nuyts RM, Felten PC, Pels E, Langerhorst CT, Geijssen HC, Grossniklaus HE et al. Histopathologic effects of mitomycin $\mathrm{C}$ after trabeculectomy in human glaucomatous eyes with persistent hypotony. Am J Ophthalmol 1994; 118: 225-237.

12 Mietz H, Brunner R, Addicks K, Krieglstein GK. Histopathology of an avascular filtering bleb after trabeculectomy with mitomycin-C. J Glaucoma 1993; 2: 266-270.

13 Sagara H, Yago K. Dry eye and bleb leaks after trabeculectomy with mitomycin C. Folia Ophthalmol Jpn 2004; 55: 18-21.
14 Tsubota K, Goto E, Fujita H, Ono M, Inoue H, Ichiro S et al. Treatment of dry eye by autologous serum application in Sjögren's syndrome. Br J Ophthalmol 1999; 83: 390-395.

15 Matsuo H, Tomidokoro A, Tomita G, Araie M. Topical application of autologous serum for the treatment of lateonset aqueous oozing or point-leak through filtering bleb. Eye 2005; 19: 23-28.

16 Izumi K, Tanino T, Ohtake Y, Kimura I, Miyata H, Mashima Y. Repair of leaking bleb with autologous serum. Atarashii Ganka 2002; 19: 669-672.

17 Mengher LS, Pandher KS, Bron AJ, Davey CC. Effect of sodium hyaluronate $(0.1 \%)$ on break-up time (NIBUT) in patients with dry eyes. $\mathrm{Br}$ J Ophthalmol 1986; 70: 442-447.

18 Norn MS. Diagnosis of dry eye. In: Lemp MA, Marqurdt R (eds). The dry eye: a comprehensive guide. Springer Verlag: Berlin, 1992, pp 133-182.

19 Salmon JF, Kanski JJ. Trabeculectomy. Glaucoma, 3rd ed. Butterworth-Heinemann: Oxford, 2004, pp 139-149.

20 Picht G, Grehn F. Classification of filtering blebs in trabeculectomy: biomicroscopy and functionality. Curr Opin Ophthalmol 1998; 9: 2-8.

21 Hu CY, Matsuo H, Tomita G, Suzuki Y, Araie M, Shirato S et al. Clinical characteristics and leakage of functioning blebs after trabeculectomy with mitomycin-C in primary glaucoma patients. Ophthalmology 2003; 110: 345-352.

22 Spalton D, Marshall J, Chong V. The normal retina, retinal imaging and the interpretation of pathological changes. In: Spalton DJ, Hitchings RA, Hunter PA, Tan JCH, Harry J (eds). Atlas of Clinical Ophthalmology, 3rd ed. ElsevierMosby: Philadelphia, 2005, pp 397-436.

23 Tsubota K, Toda I, Yagi Y, Ogawa Y, Ono M, Yoshino K. Three different types of dry eye syndrome. Cornea 1994; 13: 202-209.

24 Feenstra RP, Tseng SC. What is actually stained by Rose Bengal? Arch Ophthalmol 1992; 110: 984-993.

25 Tuft S. The cornea. In: Spalton DJ, Hitchings RA, Hunter PA, Tan JCH, Harry J (eds). Atlas of Clinical Ophthalmology, 3rd ed. Elsevier-Mosby: Philadelphia, 2005, pp 147-186.

26 Hamano T, Horimoto K, Lee M, Komemushi S. Sodium hyaluronate eye drops enhance tear film stability. Jpn J Ophthalmol 1996; 40: 62-65.

27 Watanabe H, Ohashi Y, Kinoshita S, Manabe R, Ohshiden K. Distribution of epidermal growth factor in rat ocular and periocular tissues. Graefes Arch Clin Exp Ophthalmol 1993; 231: 228-232.

28 Huang AJ, Tseng SC, Kenyon KR. Change of paracellular permeability of ocular surface epithelium by vitamin A deficiency. Invest Ophthalmol Vis Sci 1991; 32: 633-639.

29 Sugiyama T, Miyauchi S, Machida A, Miyazaki K, Tokuyasu K, Nakazawa K. The effect of sodium hyaluronate on the migration of rabbit corneal epithelium. II. The effect of topical administration. Ocul Pharmacol 1991; 7: 53-64

30 Higginbotham EJ, Stevens RK, Musch DC, Karp KO, Lichter PR, Bergstrom TJ et al. Bleb-related endophthalmitis after trabeculectomy with mitomycin C. Ophthalmology 1996; 103: 650-656.

31 Katz LJ, Cantor LB, Spaeth GL. Complications of surgery in glaucoma. Early and late bacterial endophthalmitis following glaucoma filtering surgery. Ophthalmology 1985; 92: 959-963.

$32 \mathrm{Li} \mathrm{J}$, Pang L. Influence on tear film of postoperative 5-fluorouracil and intraoperative mitomycin $\mathrm{C}$ in glaucoma filtration surgery. Zhonghua Yan Ke Za Zhi 2001; 37: 43-47. 